\title{
Development of a digital mobile solar tracker
}

\author{
Sunil Baidar ${ }^{1,2}$, Natalie Kille ${ }^{2,3}$, Ivan Ortega ${ }^{1,2}$, Roman Sinreich ${ }^{1}$, David Thomson ${ }^{2}$, James Hannigan ${ }^{4}$, and \\ Rainer Volkamer ${ }^{1,2,3}$ \\ ${ }^{1}$ Department of Chemistry and Biochemistry, University of Colorado, Boulder, CO 80309, USA \\ ${ }^{2}$ Cooperative Institute for Research in Environmental Sciences (CIRES), University of Colorado, Boulder, CO 80309, USA \\ ${ }^{3}$ Department of Atmospheric and Oceanic Sciences (ATOC), University of Colorado, Boulder, CO 80309, USA \\ ${ }^{4}$ National Center for Atmospheric Research (NCAR), Boulder, CO 80301, USA
}

Correspondence to: Rainer Volkamer (rainer.volkamer@colorado.edu)

Received: 15 September 2015 - Published in Atmos. Meas. Tech. Discuss.: 2 November 2015

Revised: 11 February 2016 - Accepted: 17 February 2016 - Published: 8 March 2016

\begin{abstract}
We have constructed and deployed a fast digital solar tracker aboard a moving ground-based platform. The tracker consists of two rotating mirrors, a lens, an imaging camera, and a motion compensation system that provides the Euler angles of the mobile platform in real time. The tracker can be simultaneously coupled to UV-Vis and Fourier transform infrared spectrometers, making it a versatile tool to measure the absorption of trace gases using solar incoming radiation. The integrated system allows the tracker to operate autonomously while the mobile laboratory is in motion. Mobile direct sun differential optical absorption spectroscopy (mobile DS-DOAS) observations using this tracker were conducted during summer 2014 as part of the Front Range Air Pollution and Photochemistry Experiment (FRAPPE) in Colorado, USA. We demonstrate an angular precision of $0.052^{\circ}$ (about $1 / 10$ of the solar disk diameter) during research drives and verify this tracking precision from measurements of the center to limb darkening (CLD, the changing appearance of Fraunhofer lines) in the mobile DS-DOAS spectra. The high photon flux from direct sun observation enables measurements of nitrogen dioxide $\left(\mathrm{NO}_{2}\right)$ slant columns with high temporal resolution and reveals spatial detail in the variations of $\mathrm{NO}_{2}$ vertical column densities (VCDs). The $\mathrm{NO}_{2}$ VCD from DS-DOAS is compared with a co-located MAX-DOAS instrument. Overall good agreement is observed amid a highly heterogeneous air mass.
\end{abstract}

\section{Introduction}

Mobile column measurements provide a means to characterize the distribution of trace gases over a large spatial scale while capturing the atmospheric variability over the column. Combined with wind measurements, mobile column measurements of trace gases have been shown to be very useful to constrain emission of trace gases from source regions by applying a mass conservation approach (e.g., Baidar et al., 2013b; Ibrahim et al., 2010; Johansson et al., 2014a, b; Mellqvist et al., 2010; Shaiganfar et al., 2011; Wang et al., 2012). Mobile column measurements from various platforms have previously been used to estimate nitrogen oxides $\left(\mathrm{NO}_{x}\right)$ emissions from cities (Baidar et al., 2013b; Ibrahim et al., 2010; Shaiganfar et al., 2011; Wang et al., 2012), nitrogen dioxide $\left(\mathrm{NO}_{2}\right)$, sulfur dioxide $\left(\mathrm{SO}_{2}\right)$ (Johansson et al., 2014a), and formaldehyde (HCHO) (Johansson et al., 2014b) from industries using the differential optical absorption spectroscopy (DOAS) (Platt and Stutz, 2008) technique and fugitive volatile organic compound (VOC) emissions from refineries (e.g., Mellqvist et al., 2010; Johansson et al., 2014a, b) using the solar occultation flux (SOF) (Mellqvist et al., 2010) method. The DOAS method typically is limited to the UV-Vis wavelength region and uses scattered sunlight; while the SOF method uses direct sun observations in the mid-IR wavelengths.

DOAS measurements of scattered sunlight are particularly attractive to conduct mobile column measurements, because scattered sunlight measurements do not require clearsky conditions and the relative ease to operate such instruments. In particular, the stability of the elevation angle (EA), 
i.e., angle relative to the horizon, is less crucial at higher EAs typically used for mobile DOAS measurements. Logistical challenges arise when measurements observe the direct solar beam from a moving platform, e.g., due to highly uncorrelated motions of vehicles on roads. However, there are advantages of using direct sun measurements also at UV-Vis wavelengths. The biggest benefit is the availability of high photon flux in the direct solar beam. This enables fast measurements (and subsequently high spatial resolution) with good signal to noise. Other advantages include (1) simple determination of the air mass factor (depends only upon the solar zenith angle) and (2) no need to account for the Ring effect (Grainger and Ring, 1962) in the DOAS analysis, which makes the retrievals more straightforward. These advantages hold potential to improve precision with direct sun measurements. In addition, extending the capabilities to measure trace gases using the direct sun observations in the UV-Vis region enables us to make simultaneous measurements of nitrogen oxides $\left(\mathrm{NO}_{x}\right)$ and VOC precursor molecules for ozone $\left(\mathrm{O}_{3}\right)$ and other secondary pollutants on similar temporal and spatial scale to study different processes in the atmosphere.

In this work we describe a fast digital mobile solar tracker that is able to motion stabilize and track the sun while moving on roads. The new tracker receives simultaneous input about the vehicle orientation from a motion compensation system and from an imaging feedback loop. This enables the tracker to autonomously locate the sun while moving and also to make measurements under thin cloud conditions. The tracker transfer optics have been designed to facilitate the flexible coupling of multiple spectrometers for direct sun absorption measurements at UV-Vis-NIR and mid-IR wavelengths. The tracker was successfully deployed from 21 July to 3 September 2014 to conduct simultaneous measurements of VOC and $\mathrm{NO}_{2}$ columns as part of the Front Range Air Pollution and Photochemistry Experiment (FRAPPE 2014) in Colorado. Figure 1 shows the schematic of the measurement principle for the instruments deployed aboard the mobile laboratory during FRAPPE 2014. In Sect. 2, we describe the instrument configuration during FRAPPE 2014 as deployed during 16 research drives (RDs). The tracker performance is characterized in Sect. 3 and is demonstrated based on tracking precision as quantified from the imaging data as well as the UVVis spectral data from RD 11 on 13 August 2014. In Sect. 4, we present $\mathrm{NO}_{2}$ vertical column density (VCD) data measured by the UV-Vis spectrometer during RD 14 on $18 \mathrm{Au}-$ gust 2014, when a MAX-DOAS instrument was co-located, which provides independent $\mathrm{NO}_{2} \mathrm{VCD}$ measurements.

\section{Instrumentation and retrievals}

\subsection{Mobile solar tracker}

The mobile solar tracker as deployed aboard the mobile laboratory is shown in Fig. 2a. An additional figure showing a

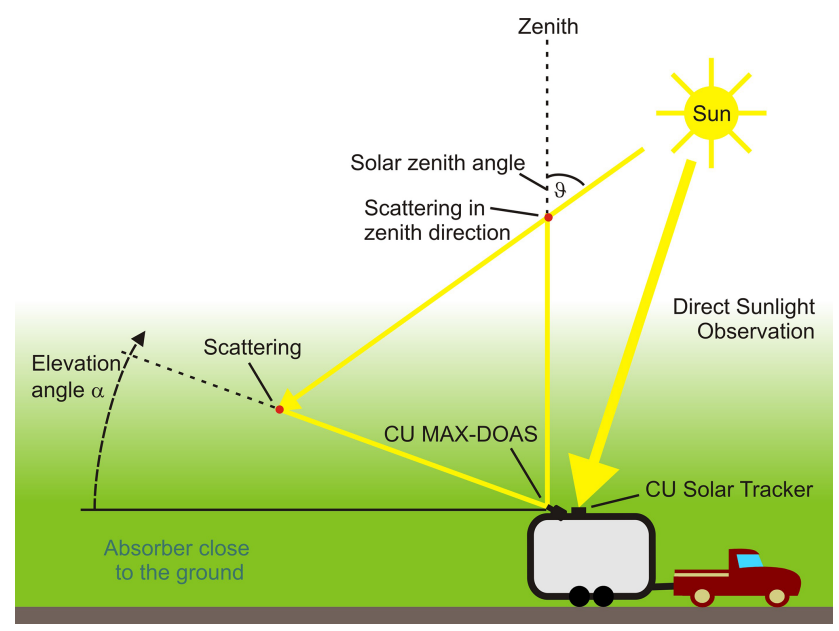

Figure 1. Illustration of measurement principle of the mobile solar tracker and the MAX-DOAS aboard the mobile laboratory deployment during FRAPPE 2014.

top and side view of the tracker is included in Supplement Fig. S1. It is an alt-azimuthal tracker consisting of two mirrors. The first mirror is mounted at a $45^{\circ}$ angle directly on a stepper motor and allows access to any EA. The direct coupling of the mirror permits fast backlash free movements. The second mirror is mounted at a $45^{\circ}$ angle opposite to the first mirror. The two mirrors are mounted on a rotational stage that is driven by a second stepper motor. The electrical connection is realized via a slip ring that transmits communication and power to the EA motor and permits unrestrained and continuous $360^{\circ}$ rotation of the mirror system. The rotational stage and the slip ring have inner (hollow axle) diameters of 2 and 1.5 inches, respectively, that transmits the solar beam. Figure $2 \mathrm{~b}$ shows the optical schematic of the solar tracker. The light is focused by a 2 inches $\mathrm{f} / 4$ lens onto an aperture plate with a $2 \mathrm{~mm}$ diameter hole. A $1.5 \mathrm{~mm}$ thick quartz diffuser plate mounted at the back of the aperture plate ensures homogeneous illumination of the UV-Vis spectrometer via a set of optical fibers. The infrared wavelength beam is directed to an IR spectrometer by using a dichroic mirror positioned at an angle of $45^{\circ}$ above the lens. The data from the IR wavelengths will be presented as part of separate publication (Kille et al., 2016).

The motion compensation system is identical to that used as part of the University of Colorado Airborne MAX DOAS (CU AMAX-DOAS) instrument (Baidar et al., 2013a; Volkamer et al., 2015). The motion compensation system is used here to correct the mirror angles for the vehicle pitch, roll, and heading in real time during the drives. Briefly, the system consists of a PC104 computer connected to the two stepper motors of the tracker, two angle sensors, a Systron Donner Inertial MMQ-G, and an electronic inclinometer. The MMQ$\mathrm{G}$ is a small robust GPS-based inertial navigation system. It provides accurate 3-D position, time, and velocity, as well as 


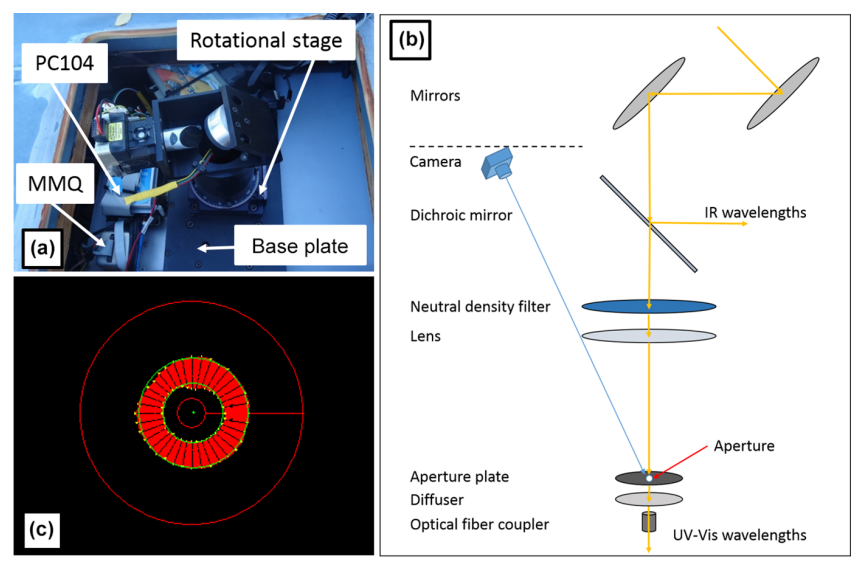

Figure 2. Instrumental setup during FRAPPE 2014. (a) The digital solar tracker mounted inside the roof hatch of the mobile laboratory; (b) optical schematic of the solar tracker; (c) processed image of the solar disk on the aperture plate showing fitted circles (green). The red circles show search region for circle fitting algorithm.

heading, pitch, and roll ( $1 \sigma=0.29^{\circ}$ from manufacturer). The pitch, roll, and heading information from the sensor is processed by custom LabVIEW software as Euler angles to calculate the astronomical solar position (Merlaud et al., 2012) relative to the real-time vehicle orientation along the drive track. The two mirror angles are then sent to the stepper motors to bring the solar disk into the field of view (FOV) of the imaging camera. Once properly initialized the motion compensation system allows for the automatized operation of the tracker during the drive.

A smart camera with an embedded PC (National Instrument $1722,480 \times 640$ pixels, $400 \mathrm{MHz}$ ) is used as an imaging feedback system to monitor and control the pointing of the tracker once the solar disk is in the FOV of the camera. The imaging camera is mounted below the baseplate of the solar tracker at an angle of $20^{\circ}$ and has a FOV of $5.2^{\circ} \times 3.9^{\circ}$. It is equipped with a standard lens with UV filter (for protection) that observes the image of the solar disk on the aperture plate. During operation, the solar disk has a diameter of about 140 pixels, corresponding to an angular resolution of about $0.0038^{\circ}$ per pixel. The $2 \mathrm{~mm}$ aperture has a diameter of about 80 pixels. The images are evaluated for the centers of the solar disk and the aperture using a built-in LabVIEW image processing algorithm to determine pixel difference between the centers of the two circles (see Fig. 2c). This code runs on the smart camera CPU in real time. The pixel offsets in $x$ and $y$ direction are determined and then sent to the PC104 via a serial data cable for fine tracking of the sun (see Fig. 2 and Sect. 2.1.1). The loop rate of the tracking system is determined by a combination of image acquisition $(\sim 20$ $30 \mathrm{~ms}$ ), offset determination by the imaging software ( $\sim 20$ $30 \mathrm{~ms}$ ), communication and angle correction (a few $\mathrm{ms}$ ), and motor latencies to execute the motor commands $(\sim 10 \mathrm{~ms})$. An overall loop rate of $15-20 \mathrm{~Hz}$ is realized. Camera-based feedback loops without access to the Euler coordinates have previously been used to precisely track the sun from a stationary setup (Gisi et al., 2011) and a research vessel (Bertleff, 2014).

\subsubsection{Tracking algorithm}

The operation of our mobile solar tracker is based on a twolevel algorithm as shown in Fig. 3. First, the real-time pitch, roll, and heading information of the platform is used as Euler angles to correct the astronomical solar position and locate the sun in the sky relative to the vehicle orientation. This calculated sun position in the local vehicular coordinate system provides the coarse mirror angles to the motors to bring the solar disk into the FOV of the camera. The solar disk images are recorded and evaluated to determine the center position of the aperture and the solar disk (Gisi et al., 2011). First, a threshold is applied to convert the image to binary format to distinguish the bright solar disk from the dark aperture plate and aperture. Next, an ellipse fitting algorithm is applied to the binary image contours. For subsequent images, depending on the distance of the solar disk to the aperture, either a circle or an ellipse fitting algorithm is applied. The circle and ellipse algorithms used here are the functions built in to the LabVIEW vision toolkit from National Instruments. The choice of ellipse vs. circle algorithm is made for efficiency, as well as due to limitations of each algorithm. The circle algorithm is significantly faster than the ellipse algorithm, as it only searches for a circle to fit within a well-defined region of the image, centered around the aperture, whereas the ellipse algorithm searches the entire camera pixel space. A consistency check is performed for the radii of fitted circles/ellipses before calculating the difference in the center position of the solar disk and aperture. Once the deviation between the aperture and the solar disk centers is determined from the camera data, a small correction is applied based on historical MMQ data to account for the motion of the platform during the control loop time, i.e., the time from when the picture was recorded and the new motor target position is commanded.

One of the major challenges with tracking the sun from a moving platform is accounting for the platform motion that happens over the course of each control loop interval. As the platform is in continuous uncorrelated motion, the orientation at the time of the recording of the image is different to that when the motor positions are updated. We used fast MMQ data at $100 \mathrm{~Hz}$ to account for this change in orientation and correct the pixel offset data from the imaging system. It was empirically determined that the correction based on the most recent data from MMQ $(t=0)$ and the data 4 points back in time $(t=-40 \mathrm{~ms})$ yielded the best result. The difference in pitch and roll angles between these two points ( $n=0$ and $n=-4)$ and the relationship between degree and pixel is used to correct the pixel offset data from the imaging system. The corrected pixel data are converted to mirror an- 


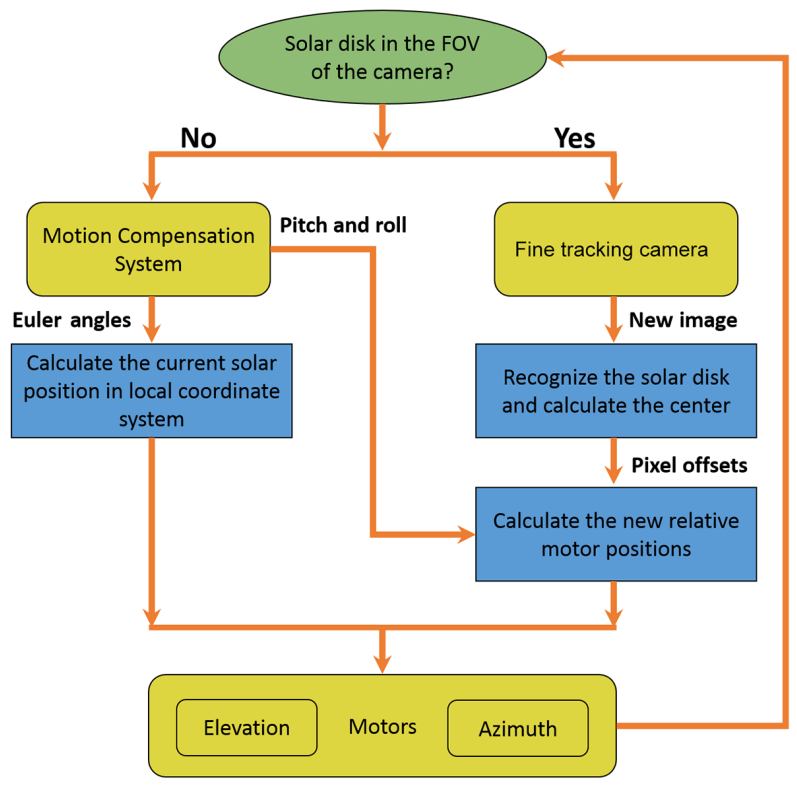

Figure 3. Flowchart of the tracking algorithm (see text for the details).

gles to update the mirror positions in order to align the two centers and subsequently keep them aligned. Application of this correction for motion of the platform leads to measurable improvements in the tracking during research drives. A cumulative density plot of percentage of points within a certain distance off of the center of the solar disk when this correction is (i) applied and (ii) not applied during segments of a research drive is shown in Fig. S2. An additional $9 \%$ of data (a relative improvement of $\sim 23 \%$ ) were found to lie in the closest bin to the center $\left(0-20\right.$ pixels, $\left.0.075^{\circ}\right)$ when the correction is applied.

\subsubsection{Advantages of integrated motion compensation system and imaging feedback}

The primary function of the motion compensation system in the mobile solar tracker setup is to accurately determine the real-time orientation of the platform in order to locate the sun in the sky while moving. The secondary purpose is to make a small correction in the imaging data in order to account for the lag time it takes to record the camera image of the solar disk, process it, and update the new motor target position. The given angle accuracy by the manufacturer for the pitch, roll, and heading from the MMQ-G is $0.29^{\circ}$. In practice, we have found that the pitch angle measured by the MMQ-G and an independent angle sensor on the NSF/NCAR GV research aircraft agree within $0.15^{\circ}(1 \sigma)$ (Baidar et al., 2013a). However, the angular diameter of the solar disk is $0.53^{\circ}$ in the sky, and the motion compensation system by itself is not good enough to track the sun accurately while moving. The imaging setup is needed for very high tracking precision and has previously been described in a ground-based stationary system (Gisi et al., 2011) and a research vessel (Bertleff, 2014). However, we have found that with the uneven motion on the road, the imaging setup alone cannot track the sun continuously during drives at any reasonable angular precision and duty cycle. By integrating the imaging setup with the motion compensation system a high level of angular precision and duty cycle is achieved. The advantages of the integrated motion compensation system and imaging feedback loop are the following:

1. The position of the sun in the sky in a wide range of situations, including obstructed and de-aligned viewing conditions, can be accurately determined. The location of the sun relative to the vehicle local orientation is known at any point in time, also when the solar disk is not in the FOV of the camera, e.g., because of bumps in the road, or trees, buildings, bridges and/or clouds obstructing the view.

2. Time needed to search for the sun in the sky is eliminated and results in an improved duty cycle of the instrument to conduct measurements and minimize data gaps.

3. Straightforward operation of the instrument is possible. Only a rough alignment is needed to orient the tracker mirrors and the motion compensation system. The final tracking accuracy is based only on the imaging loop.

4. High-precision tracking of the sun is achievable.

\subsection{Direct sun DOAS (DS-DOAS)}

The solar tracker was coupled to an Ocean Optics QE65000 spectrometer with thermo-electrically cooled charge coupled device (CCD) array detector via optical fiber bundles for DSDOAS measurements. A $12 \mathrm{~m}$ long $1.5 \mathrm{~mm}$ diameter fiber bundle transfers the direct solar beam from the solar tracker to a $10 \mathrm{~m}$ long $1.7 \mathrm{~mm}$ diameter single core silica fiber to minimize polarization effects. The other end of the monofiber is connected to a fiber bundle that delivers light to a single UV-Vis spectrometer, or to a bifurcated fiber bundle connected to two or more UV-Vis spectrometers. The mono-fiber was found to be critical during the DOAS analysis of the UV-Vis spectra. Without the mixing mono-fiber the root mean square of the DOAS fit increased gradually over a short period of time, which was not observed when the mixing fiber was in place. A single spectrometer that covered the wavelength range of 390 to $520 \mathrm{~nm}$ with $\sim 0.55 \mathrm{~nm}$ resolution (full width at half maximum) was used during FRAPPE 2014 to measure $\mathrm{NO}_{2}$ VCDs. The optical spectrometer bench was heated and kept constant at a temperature of $40 \pm 0.05^{\circ} \mathrm{C}$, to minimize changes in optical properties, while the detector itself was cooled to $-10^{\circ} \mathrm{C}$ to reduce dark current. The temperature stability was maintained by using a two-stage temperature-controlled housing described in 
Coburn et al. (2011). The integration time for each spectrum was $2 \mathrm{~s}$. An absorptive neutral density filter with an optical density of 1.6 (e-based) was placed above the focusing lens of the solar tracker to avoid saturation of the detector.

\subsection{Mobile multi-axis DOAS (MAX-DOAS)}

The MAX-DOAS instrument uses scattered sunlight as the light source. The collected spectra are then analyzed for trace gases such as $\mathrm{NO}_{2}$, glyoxal, $\mathrm{HCHO}$, and $\mathrm{O}_{4}$ using the DOAS method. The MAX-DOAS instrument is described in detail in Sinreich et al. (2010) and Coburn et al. (2011). Briefly, the MAX-DOAS telescope was mounted on the roof of the mobile laboratory next to the solar tracker and collected scattered photons at different EAs. Spectra collected from different EAs contain information from different layers in the atmosphere and hence can be used to obtain information about the vertical distribution of the trace gases. However, due to the horizontal gradient of the trace gases of interest, MAXDOAS measurements from a mobile platform are focused on obtaining VCDs using one or two EAs (e.g., Ibrahim et al., 2010; Shaiganfar et al., 2011; Wang et al., 2012). During FRAPPE 2014, the MAX-DOAS instrument made measurements at $30^{\circ}$ (facing backward relative to the mobile platform) and $90^{\circ}$ (zenith) EAs. The collected photons are transferred to a spectrometer/CCD detector system via an optical glass fiber bundle. The measured spectra at $30^{\circ}$ EA were analyzed for $\mathrm{NO}_{2}$ using a zenith reference spectrum, and the resulting differential slant column densities (dSCD) were converted into VCDs using the geometric air mass factor (which is 2 for $30^{\circ} \mathrm{EA}$ ).

\subsection{Data retrievals}

\subsubsection{DOAS analysis}

The wavelength range of $434-460 \mathrm{~nm}$ was used for DOAS retrieval of $\mathrm{NO}_{2}$. Trace gas reference cross sections for $\mathrm{NO}_{2}$ at $298 \mathrm{~K}$ (Vandaele et al., 1998), orthogonal $\mathrm{NO}_{2}$ at $220 \mathrm{~K}$, $\mathrm{O}_{3}$ (Serdyuchenko et al., 2014), $\mathrm{H}_{2} \mathrm{O}$ (Rothman et al., 2013), $\mathrm{O}_{4}$ (Thalman and Volkamer, 2013), glyoxal (Volkamer et al., 2005), and a center to limb darkening (CLD) reference spectrum (Bosch et al., 2003) were simultaneously fitted using a nonlinear least square fitting routine. The CLD correction reference spectrum was calculated as described in Bosch et al. (2003) and fitted to account for uneven decreases in intensity of solar spectrum at the center and at the limb of the solar disk. CLD is also used to evaluate tracker performance (see Sect. 2.4.2). A fifth-order polynomial to account for scattering processes and broadband absorption in the atmosphere as well as broadband instrumental features, and an additional intensity offset to account for instrument stray light were also included in the fitting procedure. A spectrum from a clean background region was included as the Fraunhofer reference spectrum in the analysis. Figure $\mathrm{S} 3$ shows a spectral fit for $\mathrm{NO}_{2}$ and the corresponding residual from the DOAS fit.

The MAX-DOAS analysis used the same settings as the DS-DOAS analysis. The only difference is that a Ring spectrum was used to correct for the Ring effect in place of the CLD reference spectrum.

\subsubsection{Center to limb darkening (CLD)}

The effective emission temperature for solar radiation coming from the center is higher compared to the edges of the solar disk. This decrease in effective emission temperature results in an observed decrease in solar intensity towards the edges of the solar disk. This effect is known as the centerto-limb darkening (Hestroffer and Magnan, 1998). The optical depth (OD) of the solar Fraunhofer lines also decreases from the center to the edges of the solar disk and results in the need for the CLD correction in DOAS analysis for UVVis solar occultation measurements (e.g., Bosch et al., 2003; Butz, 2006; Gill et al., 2000). An empirical approach to correct for the CLD effect was developed by Bosch et al. (2003) and showed that the addition of a CLD correction improved DOAS retrieval of iodine monoxide (IO) and chlorine dioxide $(\mathrm{OClO})$ from balloon-borne solar occultation measurements. High-resolution solar spectra taken from the solar disk center and averaged over the solar disk were used to create a reference pseudo-absorber cross section that was included in the DOAS fits (spectra available at http://www.hs. uni-hamburg.de/DE/Oef/Inf/Einbl/Sospec/sonnspec.html).

We evaluated the UV-Vis spectra in the wavelength window from 400 to $440 \mathrm{~nm}$ for the CLD correction fit coefficient to estimate the effective tracking precision from spectral data. This fit window was chosen to include two strong Fraunhofer lines ( $\mathrm{H} \delta$ at $410.2 \mathrm{~nm}$ and $\mathrm{H} \gamma$ at $434.0 \mathrm{~nm}$ ) in the DOAS analysis. For radiation from the central portion of the solar disk we expect near-zero CLD. We expect a significant CLD signal when the pointing accuracy is suboptimal, i.e., when radiation from near the edges of the solar disk contributes significantly to the overall photon flux of our UV-Vis spectra. Figure 4 shows the spectral proofs of CLD correction fits for spectrum taken at the center of the solar disk, 25 pixels $\left(0.095^{\circ}\right)$ off of the center, and 50 pixels $\left(0.19^{\circ}\right)$ off of the center. Figure 4 clearly illustrates that the CLD correction becomes more important as spectra are collected further away from the center of the solar disk. The inclusion of CLD reference spectrum in the retrieval (1) improved $\mathrm{NO}_{2}$ fit, (2) minimized residuals, and (3) reduced scattering in the retrieved $\mathrm{NO}_{2}$ slant columns. 

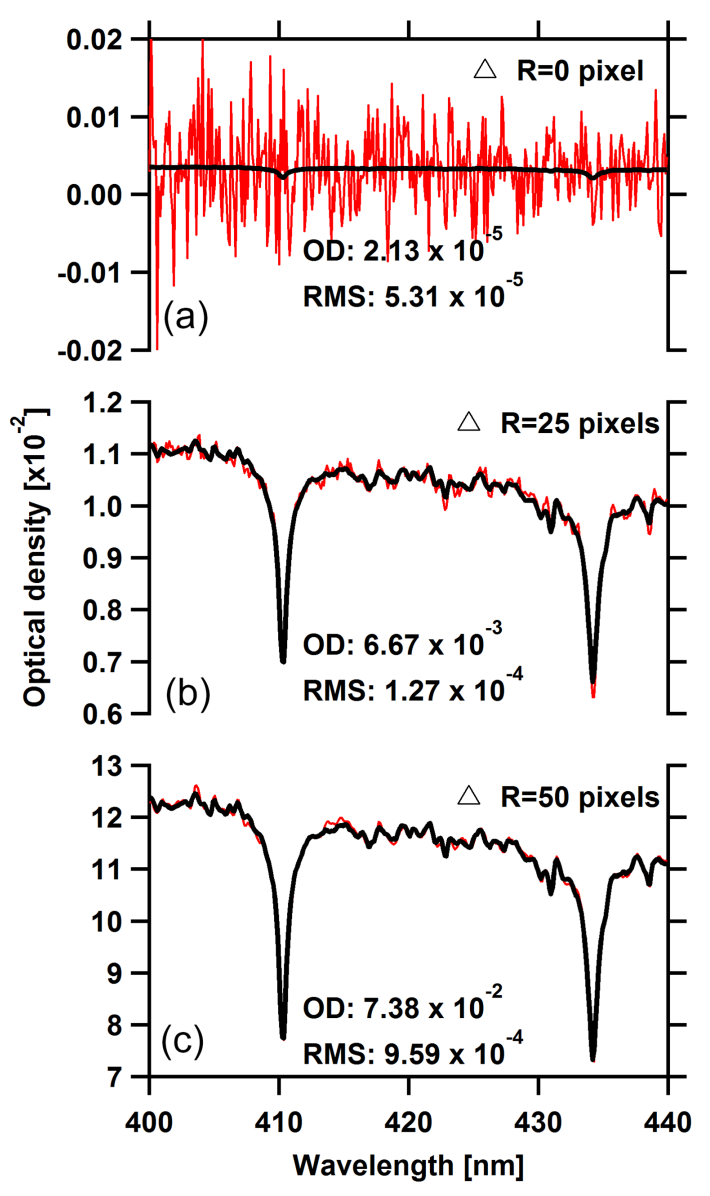

Figure 4. Spectral fits of CLD reference for spectra taken at 0,25 $\left(0.095^{\circ}\right)$ and 50 pixels $\left(0.19^{\circ}\right)$ off of the center of the solar disk. The optical density of the CLD fit result and the root-mean-square residual that remains after subtracting all absorbers are also shown.

\section{Results and discussion}

\subsection{Evaluation of the tracker accuracy from the imaging feedback}

The pointing accuracy of the solar tracker was determined from the deviation of the center of the two fitted circles (i.e., aperture and solar disk) shown in Fig. 2c. The deviation in $x$ and $y$ direction in camera FOV was converted to angular deviation using the relationship between the diameter of the sun in the camera picture and its angular diameter in the sky. The locations of the center of the solar disk for every circle/ellipse fit from the RD 11 (duration $\sim 8 \mathrm{~h}$ ) on 13 August 2014 are shown in Fig. S4. Figure 5 shows two histograms of the angular deviation between the centers of the two circles in $x$ and $y$ direction during the RD 11 (only data taken when the vehicle was in motion are included). The $1 \sigma$ standard deviation in $x$ and $y$ direction determined from a Gaussian fit (red lines) are 0.035 and $0.039^{\circ}$ respectively giving us an overall pointing precision of $0.052^{\circ}$. Hence the pointing precision $(1 \sigma)$ of about $1 / 10$ of the solar disk angular diameter in the sky was achieved during mobile operation. Note that data points when the solar disk was not in the FOV of the camera are excluded from this analysis. On average the solar disk ventured outside the FOV of the camera every 3-4 s but was highly variable. The solar disk was in the FOV of the camera $88 \%$ of the time that the mobile laboratory was in motion. Of the $\sim 12 \%$ that the system needed to reacquire the solar disk, the sun was back in the FOV within $0.5 \mathrm{~s}$ for $90 \%$ of the instances that it was lost. The system is largely unaffected by these short losses because of the fast scan acquisition time $(8 \mathrm{~ms})$, which enables filtering of good data with $8 \mathrm{~ms}$ time resolution. A $2 \mathrm{~s}$ spectrum accumulates many $8 \mathrm{~ms}$ scans, and scans that do not meet a minimum threshold signal criteria are automatically eliminated. Thus the $2 \mathrm{~s}$ spectra contain useful data that can be evaluated for $\mathrm{NO}_{2}$ VCDs with a duty cycle of $\sim 91 \%$ of the drive time under clear-sky conditions.

Figure $6 \mathrm{a}$ and $\mathrm{b}$ show the box plot of angular deviation as a function of vehicular speed and solar zenith angle (SZA) respectively. The top and bottom of the box represent the 25th and 75th percentile of the data respectively while the middle line is the median. The red lines indicate the $1 \sigma$ tracking precision from the Gaussian fits (Fig. 5). The distribution appears to be slightly larger at larger speeds, and above $50^{\circ} \mathrm{SZA}$; however, the means were not found to be statistically different. The apparent slight losses in precision seen at $5-15 \mathrm{~km} \mathrm{~h}^{-1}$ and $20-30^{\circ}$ SZA were also not statistically different from the mean. Also, this behavior is not observed during other drives. Future improvement in the tracker could involve incorporating a faster camera and motor in order to decrease the control loop time. Note that a much higher precision tracking $\left(<0.005^{\circ}\right)$ is achieved in the stationary mode, which is not affected by the control loop time.

\subsection{Evaluation of the tracker accuracy from the CLD}

Figure S5 shows CLD fit coefficient (absolute value) as a function of distance from the center. The chosen Fraunhofer reference spectrum from the center of the solar disk for the DOAS analysis does not always have the highest OD for the Fraunhofer lines since it also depends upon the SZA. Thus, depending upon the SZA of the reference spectrum and the measured spectrum, the CLD fit coefficient can change sign and require the use of absolute value. The fit coefficient gradually increases with the increasing distance from the center of the disk until it is 25-30 pixels off of the center. After that the increase in the fit coefficient is much more pronounced. In fact, from 0 to 30 pixels the CLD fit coefficient is not very sensitive to the pixel offset. This is because CLD is a power law function of distance from the center of the solar disk to the limb and hence is not very sensitive close to the center (Hestroffer and Magnan, 1998). This insensitivity closer to the center is likely further pronounced in our setup for the following reasons: (1) the size of the aperture used to collect the radiation for the UV-Vis spectra $(\sim 40$ pixels 


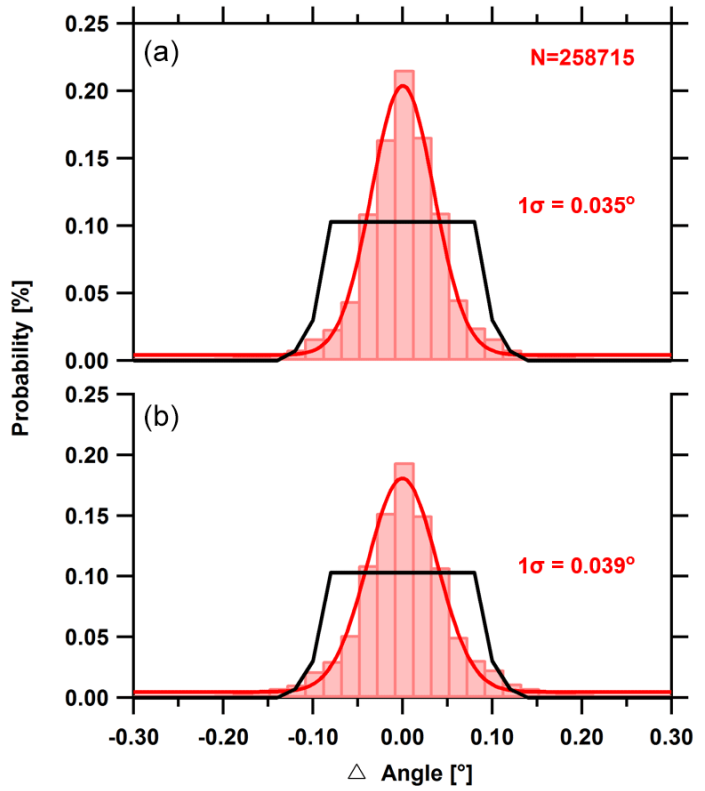

Figure 5. Pointing precision during the RD 11 on 13 August 2014. Distributions of the deviations between the solar disk center and the aperture center in (a) $x$ direction and (b) $y$ direction. The red lines show the Gaussian fits to the data. The black lines represent the tracking precision derived from CLD fits. The flat top of the distribution reflects the fact CLD signals are significant only at the edges of the solar disk (see Fig. S5 for details).

in radius) results in radiance-weighted average spectra that show no significant need for CLD correction until it is 25-30 pixels off of the center. (2) As the SZA of the sun changes the observed CLD is a combination of the solar movement and pointing inaccuracy and can have compensating effects over small scales. It is clearly evident from Fig. S5 that the CLD ODs from DOAS fits are a robust method of quantitatively determining whether an individual spectrum was taken within 30 pixels of the center or outside this threshold. The CLD ODs from the DOAS analysis for the RD 11 are shown in Fig. S6 as a histogram and a cumulative probability distribution function for different fit coefficient bins. Figure S6 shows that $95 \%$ of the data are within the CLD OD of 0.13 , which corresponds to pixel offset of $\sim 30$ pixels or an angular precision of about $0.12^{\circ}(2 \sigma)$. This is consistent with angular tracking precision of $0.052^{\circ}(1 \sigma)$ determined from the camera data. The black lines in Fig. 5 represent the tracking precision derived from CLD fits. The flat top of the distribution reflects the fact that CLD is insensitive across the solar disk and only at the edges the CLD signals are significant (see Fig. S5 for details). The precision of the CLD correction fits from DOAS analysis has been crosschecked using the CLD correction fit from the $\mathrm{NO}_{2}$ retrieval window and showed excellent agreement (slope $=1.07$, intercept $=1.77 \times 10^{-3}$, $R^{2}=0.97$; see Fig. S7). The $7 \%$ increase in slope is very likely due to the wavelength dependence of CLD. A $9 \pm 3 \%$

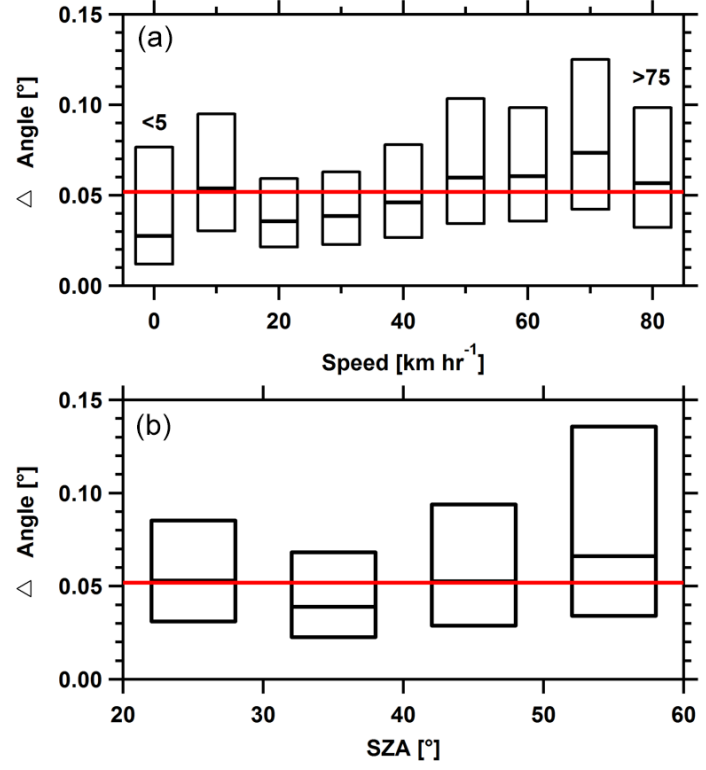

Figure 6. Pointing precision as a function of (a) vehicle speed, and (b) solar zenith angle during the RD 11 on 13 August 2014. The top, center, and bottom of the box represent 75th, 50th (median), and 25th percentiles of the data. The red lines represent the overall $1 \sigma$ pointing precision from the Gaussian fits (Fig. 5).

increase in CLD fit coefficient is expected based on the wavelength dependence from 420 to $450 \mathrm{~nm}$ (Hestroffer and Magnan, 1998).

\subsection{Field applications: comparison with MAX-DOAS}

$\mathrm{NO}_{2}$ VCDs measured by DS-DOAS using the mobile solar tracker were compared with VCDs from a co-located MAXDOAS instrument on our mobile laboratory. $\mathrm{NO}_{2} \mathrm{dSCD}$ from both instruments were converted into VCDs using a geometric air mass factor $\left(\right.$ geoAMF $\left.=\frac{1}{\sin (\mathrm{EA})}\right)$. Figure $7 \mathrm{~b}$ shows the time series of $\mathrm{NO}_{2}$ VCDs measured by the two instruments during RD 14 on 18 August 2014 in northern Colorado. The drive track for the research drive is shown in Fig. S8. The $\mathrm{NO}_{2}$ detection limit and precision $(1 \sigma)$ for the direct sun measurements are $7 \times 10^{14}$ and $3 \times 10^{14}$ molecules $\mathrm{cm}^{-2}$ respectively for a $2 \mathrm{~s}$ integration time. Even though the two instruments are co-located and used a similar retrieval, the comparison is not straightforward because of the following: (1) the viewing geometries of the two instruments are not the same. MAX-DOAS measurements were made at $30^{\circ}$ EA facing towards the back of the mobile laboratory. DS-DOAS observations depend upon the solar elevation and azimuth angle $(\mathrm{Az})$ at the time of the measurements. Thus, the two instruments are typically not looking at the same air mass. (2) Both instruments make measurements averaged horizontally over a distance that depends upon the EA. For example, for a boundary layer height of $1 \mathrm{~km}$, the MAX-DOAS observations at $30^{\circ} \mathrm{EA}$ average over a horizontal distance 
of $1.7 \mathrm{~km}$ (geometric path). In contrast, the direct sun observation only averages over a horizontal distance of $0.6 \mathrm{~km}$ at solar elevation of $60^{\circ}$. The EA and $\mathrm{Az}$ angles at the time of measurements for the two instruments are shown in Fig. 7a. As expected, the agreement between $\mathrm{NO}_{2} \mathrm{VCDs}$ is found to be best when the two instruments have similar viewing geometry. An expanded view of $\mathrm{NO}_{2} \mathrm{VCD}$ over two 9 min periods when (i) EA and $\mathrm{Az}$ angles for the two instrument are relatively similar (i.e., looking at the same air mass; $\delta \mathrm{EA}=\sim 10^{\circ}$ and $\delta \mathrm{Az}=\sim 70^{\circ}$ ) and (ii) difference in EA and $\mathrm{Az}$ angles are larger (i.e., looking at the different air masses; $\delta \mathrm{EA}=\sim 25^{\circ}$ and $\delta \mathrm{Az}=\sim 140^{\circ}$ ) are shown in Fig. $7 \mathrm{c}$ and $\mathrm{d}$ respectively. The agreement between the two instruments is indeed better in Fig. 7c compared to Fig. 7d. During the period shown in Fig. 7d, the two instruments were almost looking in opposite directions along the drive track. As the MAXDOAS was looking towards the back of the mobile laboratory, it observes the air mass probed by the solar tracker after a certain time which is dependent upon the speed of the vehicle. Figure $7 \mathrm{~d}$ shows a small offset in the magnitude of $\mathrm{NO}_{2}$ VCD as well as time (see peak at 17:37). The small offset in magnitude is likely due the difference in EA where the MAX-DOAS averages over a larger distance while the offset in time is a result of Az viewing geometry.

Despite these complications, the time series for $\mathrm{NO}_{2}$ measured by the two instruments track each other very well. High photon flux in the direct solar beam enabled fast measurements with high signal to noise, and this is evident in the time series. The DS-DOAS measurements captured variability in $\mathrm{NO}_{2}$ at much finer scale than the MAX-DOAS data. This has potentially important benefits with resolving column enhancements of spatially confined emission sources, and the spatial variations within plumes.

The good agreement between the DS-DOAS and MAXDOAS observations is reflected in the slope of $0.97 \pm 0.03$ for the orthogonal distance regression of the two data sets (Fig. S9, offset $=-1.1 \pm 1.3 \times 10^{14}$ molecules $\mathrm{cm}^{-2}$ ). All data shown in Fig. 7b are included in the comparison regardless of the EA and azimuth angle difference. The DS-DOAS data are averaged for $20 \mathrm{~s}$ to the MAX-DOAS timestamp. If the data are filtered for the solar EA (i) below $45^{\circ}$ and (ii) above $45^{\circ}$, the slopes of the orthogonal least squares fits improve to unity ( $1.01 \pm 0.04$ for case (i) and $1.00 \pm 0.04$ for case (ii)) but not significantly different from the fit to all data. The offset is larger $\left(-5.0 \pm 1.7 \times 10^{14}\right.$ molecules $\left.\mathrm{cm}^{-2}\right)$ for the second case. Such good agreement gives confidence in the validity of the new solar tracker measurements for $\mathrm{NO}_{2}$ and other species using the DS-DOAS method. The data will be used to quantify emissions of trace gases from various sources such as power plants, refineries, farms, and feedlots and will be part of subsequent publications.

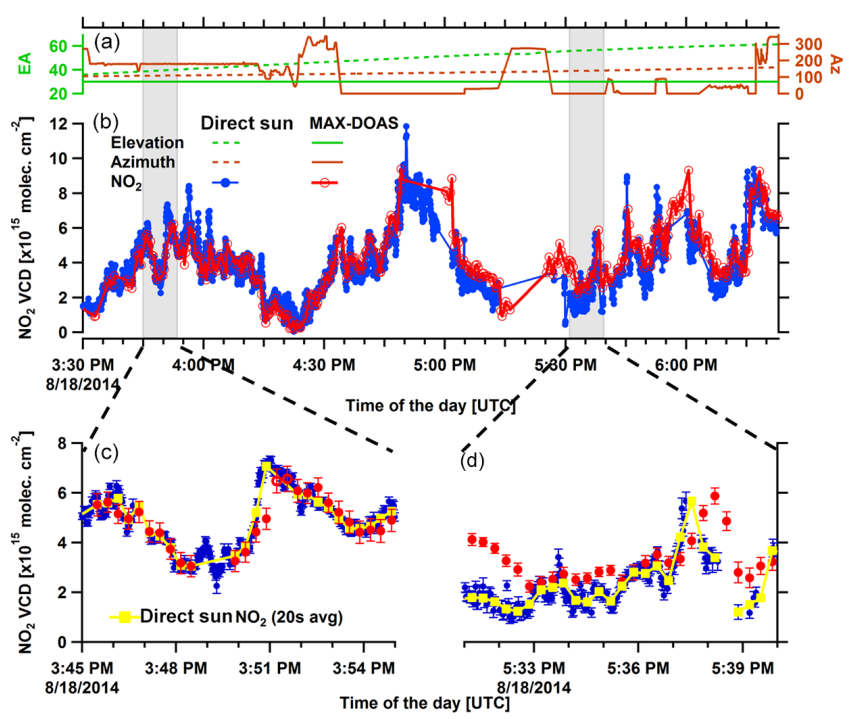

Figure 7. Measurements of $\mathrm{NO}_{2} \mathrm{VCD}$ aboard the mobile laboratory during the RD 14 on 18 August 2014 in northern Colorado. (a) The EA (green) and the Az (brown) angles for the DS-DOAS (dashed) and MAX-DOAS (solid). (b) Time series of $\mathrm{NO}_{2} \mathrm{VCD}$ measured by the DS-DOAS (blue) and the MAX-DOAS (red). Panels (c) and (d) show expansion into two 9 min period (grey boxes) when (i) difference in EA and Az between the two instruments was $\sim 10^{\circ}$ and $\sim 70^{\circ}$ and (ii) difference in EA and Az was $\sim 25^{\circ}$ and $\sim 140^{\circ}$. The yellow line represents the DS-DOAS data averaged to the MAX-DOAS timestamp. Note that the MAX-DOAS instrument was acquiring a zenith reference spectrum at 15:49 for $60 \mathrm{~s}$. The open red circles in panel (c) represent the MAX-DOAS data point which did not pass the root-mean-square-based quality control but was included for comparison purposes. The error bars in panel (c) and (d) represent $1 \sigma$ fit errors.

\section{Conclusion and outlook}

A digital solar tracker design is presented that meets the criteria to track the sun from a moving platform such as a groundbased mobile laboratory. It provides access to the solar beam with a pointing precision of $0.052^{\circ}$ from a platform that is in motion. The effective pointing precision was verified using CLD measurements. These characteristics have proven to be adequate to acquire high-resolution atmospheric absorption spectra with a high signal-to-noise ratio at UV-Vis wavelengths. $\mathrm{NO}_{2} \mathrm{VCDs}$ were measured with the mobile solar tracker (DS-DOAS) and compared with VCDs from a colocated MAX-DOAS instrument. A good overall agreement is found amid a highly inhomogeneous environment. In the future, the setup provides potential for 1-2 orders of magnitude higher photon intensity that could be exploited to maximize the signal-to-noise ratio to measure trace gases at UV wavelengths (e.g., HONO, HCHO), to conduct nighttime atmospheric measurements with the moon as a light source, or to couple multiple spectrometers to observe other trace gases at UV-Vis-NIR or mid-IR wavelengths. 


\section{The Supplement related to this article is available online at doi:10.5194/amt-9-963-2016-supplement.}

Acknowledgements. This work was supported by an Energy Initiative Seed Grant from CIRES and Colorado Department for Public Health and the Environment (CDPHE) contract no. 14FAA64390 (Rainer Volkamer). Sunil Baidar is a recipient of an ESRL-CIRES Graduate Fellowship. The authors thank Jim Kastengren and CIRES Instrument shop for instrument design and fabrication, Philip Handley, and Owen Cooper for support during FRAPPE, Andre Butz for helpful discussions, and Caroline Fayt and Michel van Roozendael for the WinDOAS software.

Edited by: D. Feist

\section{References}

Baidar, S., Oetjen, H., Coburn, S., Dix, B., Ortega, I., Sinreich, R., and Volkamer, R.: The CU Airborne MAX-DOAS instrument: vertical profiling of aerosol extinction and trace gases, Atmos. Meas. Tech., 6, 719-739, doi:10.5194/amt-6-719-2013, 2013a.

Baidar, S., Volkamer, R., Alvarez II, R. J., Brewer, W. A., Davies, F., Langford, A. O., Oetjen, H., Pearson, G., Senff, C. J., and Hardesty, R. M.: Combining active and passive airborne remote sensing to quantify $\mathrm{NO}_{2}$ and $\mathrm{O}_{x}$ production near Bakersfield, CA, British Journal of Environment and Climate Change, 3, 566586, doi:10.9734/BJECC/2013/5740, 2013b.

Bertleff, M.: Camera based sun tracking system for mobile platforms, Masters thesis, Karlsruhe Institute of Technology, Germany, 2014

Bosch, H., Camy-Peyret, C., Chipperfield, M. P., Fitzenberger, R., Harder, H., Platt, U., and Pfeilsticker, K.: Upper limits of stratospheric IO and OIO inferred from center-to-limb-darkeningcorrected balloon-borne solar occultation visible spectra: Implications for total gaseous iodine and stratospheric ozone, J. Geophys. Res.-Atmos., 108, 4455, doi:10.1029/2002JD003078, 2003.

Butz, A.: Case studies of stratospheric nitrogen, chlorine and iodine photochemistry based on balloon borne UV/Vis and IR absorption spectroscopy, $\mathrm{PhD}$ dissertation, Ruperto Carola University of Heidelberg, Germany, 2006.

Coburn, S., Dix, B., Sinreich, R., and Volkamer, R.: The CU ground MAX-DOAS instrument: characterization of RMS noise limitations and first measurements near Pensacola, FL of BrO, IO, and CHOCHO, Atmos. Meas. Tech., 4, 2421-2439, doi:10.5194/amt-4-2421-2011, 2011.

Gil, M., Puentedura, O., Yela, M., and Cuevas, E.: Behavior of $\mathrm{NO}_{2}$ and $\mathrm{O}_{3}$ columns during the eclipse of February 26, 1998, as measured by visible spectroscopy, J. Geophys. Res.-Atmos., 105, 3583-3593, doi:10.1029/1999JD900973, 2000.

Gisi, M., Hase, F., Dohe, S., and Blumenstock, T.: Camtracker: a new camera controlled high precision solar tracker system for FTIR-spectrometers, Atmos. Meas. Tech., 4, 47-54, doi:10.5194/amt-4-47-2011, 2011.

Grainger, J. and Ring, J.: Anomalous Fraunhofer Line Profiles, Nature, 193, p. 762, doi:10.1038/193762a0, 1962.
Hestroffer, D. and Magnan, C.: Wavelength dependency of the Solar limb darkening, Astronom. Astrophys., 333, 338-342, 1998.

Ibrahim, O., Shaiganfar, R., Sinreich, R., Stein, T., Platt, U., and Wagner, T.: Car MAX-DOAS measurements around entire cities: quantification of $\mathrm{NO}_{\mathrm{x}}$ emissions from the cities of Mannheim and Ludwigshafen (Germany), Atmos. Meas. Tech., 3, 709-721, doi:10.5194/amt-3-709-2010, 2010.

Johansson, J. K. E., Mellqvist, J., Samuelsson, J., Offerle, B., Lefer, B., Rappenglueck, B., Flynn, J., and Yarwood, G.: Emission measurements of alkenes, alkanes, $\mathrm{SO}_{2}$, and $\mathrm{NO}_{2}$ from stationary sources in southeast Texas over a 5 year period using SOF and mobile DOAS, J. Geophys. Res.-Atmos., 119, 1973-1991, doi:10.1002/2013JD020485, 2014a.

Johansson, J. K. E., Mellqvist, J., Samuelsson, J., Offerle, B., Moldanova, J., Rappenglueck, B., Lefer, B., and Flynn, J.: Quantitative measurements and modeling of industrial formaldehyde emissions in the Greater Houston area during campaigns in 2009 and 2011, J. Geophys. Res.-Atmos., 119, 4303-4322, doi:10.1002/2013JD020159, 2014b.

Kille, N., Baidar, S., Sinreich, R., Ortega, I., Cooper, O., Hannigan, J., Hase, F., and Volkamer, R.: The CU Mobile Solar Occultation Flux instrument: measurements of $\mathrm{NH}_{3}, \mathrm{C}_{2} \mathrm{H}_{6}, \mathrm{HCHO}$ and $\mathrm{NO}_{2}$, in preparation, 2016.

Mellqvist, J., Samuelsson, J., Johansson, J., Rivera, C., Lefer, B., Alvarez, S., and Jolly, J.: Measurements of industrial emissions of alkenes in Texas using the solar occultation flux method, J. Geophys. Res.-Atmos., 115, D00F17, doi:10.1029/2008JD011682, 2010.

Merlaud, A., De Maziere, M., Hermans, C., and Cornet, A.: Equations for Solar Tracking, Sensors, 12, 4074-4090, doi:10.3390/s120404074, 2012.

Platt, U. and Stutz, J.: Differential Optical Absorption Spectroscopy: Principles and Applications, Springer Verlag, Heidelberg, 2008

Rothman, L. S., Gordon, I. E., Babikov, Y., Barbe, A., Benner, D. C., Bernath, P. F., Birk, M., Bizzocchi, L., Boudon, V., Brown, L. R., Campargue, A., Chance, K., Cohen, E. A., Coudert, L. H., Devi, V. M., Drouin, B. J., Fayt, A., Flaud, J., Gamache, R. R., Harrison, J. J., Hartmann, J.-M., Hill, C., Hodges, J. T., Jacquemart, D., Jolly, A., Lamouroux, J., Le Roy, R. J., Li, G., Long, D. A., Lyulin, O. M., Mackie, C. J., Massie, S. T., Mikhailenko, S., Mueller, H. S. P., Naumenko, O. V., Nikitin, A. V., Orphal, J., Perevalov, V., Perrin, A., Polovtseva, E. R., Richard, C., Smith, M. A. H., Starikova, E., Sung, K., Tashkun, S., Tennyson, J., Toon, G. C., Tyuterev, Vl G., and Wagner, G.: The HITRAN 2012 molecular spectroscopic database, J. Quant. Spectrosc. Ra., 130, 4-50, doi:10.1016/j.jqsrt.2013.07.002, 2013.

Serdyuchenko, A., Gorshelev, V., Weber, M., Chehade, W., and Burrows, J. P.: High spectral resolution ozone absorption crosssections - Part 2: Temperature dependence, Atmos. Meas. Tech., 7, 625-636, doi:10.5194/amt-7-625-2014, 2014.

Shaiganfar, R., Beirle, S., Sharma, M., Chauhan, A., Singh, R. P., and Wagner, T.: Estimation of $\mathrm{NO}_{x}$ emissions from Delhi using Car MAX-DOAS observations and comparison with OMI satellite data, Atmos. Chem. Phys., 11, 10871-10887, doi:10.5194/acp-11-10871-2011, 2011.

Sinreich, R., Coburn, S., Dix, B., and Volkamer, R.: Ship-based detection of glyoxal over the remote tropical Pacific Ocean, 
Atmos. Chem. Phys., 10, 11359-11371, doi:10.5194/acp-1011359-2010, 2010.

Thalman, R. and Volkamer, R.: Temperature dependent absorption cross-sections of $\mathrm{O}_{2}-\mathrm{O}_{2}$ collision pairs between 340 and $630 \mathrm{~nm}$ and at atmospherically relevant pressure, Phys. Chem. Chem. Phys., 15, 15371-15381, doi:10.1039/c3cp50968k, 2013.

Vandaele, A., Hermans, C., Simon, P., Carleer, M., Colin, R., Fally, S., Merienne, M., Jenouvrier, A., and Coquart, B.: Measurements of the NO2 absorption cross-section from $42000 \mathrm{~cm}^{-1}$ to $10000 \mathrm{~cm}^{-1}(238-1000 \mathrm{~nm})$ at $220 \mathrm{~K}$ and $294 \mathrm{~K}$, J. Quant. Spectrosc. Ra., 59, 171-184, doi:10.1016/S0022-4073(97)00168-4, 1998.

Volkamer, R., Spietz, P., Burrows, J., and Platt, U.: Highresolution absorption cross-section of glyoxal in the UV-vis and IR spectral ranges, J. Photoch. Photobio. A, 172, 35-46, doi:10.1016/j.jphotochem.2004.11.011, 2005.
Volkamer, R., Baidar, S., Campos, T. L., Coburn, S., DiGangi, J. P., Dix, B., Eloranta, E. W., Koenig, T. K., Morley, B., Ortega, I., Pierce, B. R., Reeves, M., Sinreich, R., Wang, S., Zondlo, M. A., and Romashkin, P. A.: Aircraft measurements of $\mathrm{BrO}$, $\mathrm{IO}$, glyoxal, $\mathrm{NO}_{2}, \mathrm{H}_{2} \mathrm{O}, \mathrm{O}_{2}-\mathrm{O}_{2}$ and aerosol extinction profiles in the tropics: comparison with aircraft-/ship-based in situ and lidar measurements, Atmos. Meas. Tech., 8, 2121-2148, doi:10.5194/amt-8-2121-2015, 2015.

Wang, S., Zhou, B., Wang, Z., Yang, S., Hao, N., Valks, P., Trautmann, T., and Chen, L.: Remote sensing of $\mathrm{NO}_{2}$ emission from the central urban area of Shanghai (China) using the mobile DOAS technique, J. Geophys. Res.-Atmos., 117, D13305, doi:10.1029/2011JD016983, 2012. 